\title{
Schnelle Hilfe für trockene, sehr trockene und atopische Haut
}

\begin{abstract}
Mit Atoderm hat BIODERMA eine Hautpflege entwickelt, die auf die speziellen Bedürfnisse der trockenen, sehr trockenen und atopischen Haut eingeht. Von der Reinigung bis zur täglichen Pflege sind alle Atoderm-Produkte so aufeinander abgestimmt, dass sie die Symptome der trockenen Haut schnell lindern und ihre Widerstandskraft gegen äußere Reize stärken.
\end{abstract}

Bei trockener Haut ist der Hydrolipidfilm nicht mehr komplett intakt und die Lipidsynthese ist gestört. Wichtige Feuchtigkeit verdunstet regelrecht, da die schützende Schicht sie nicht mehr in der Dermis und Epidermis zurückhält. Je stärker die Haut austrocknet, desto leichter wird es für Allergene, Umweltgifte und schädigende Stoffe, in die Haut einzudringen. Der Effekt: Die Haut ist irritiert und gereizt, brennt und ist Aggressoren nahezu schutzlos ausgeliefert. Im schlimmsten Fall bilden sich sogar Ekzeme. Zudem weist trockene Haut einen veränderten $\mathrm{pH}$-Wert auf, der pathologischen, krank machenden Mikroorganismen einen optimalen Lebensraum bietet. Trockene Haut hat noch einen deutlich sichtbaren Neben- effekt: da es ihr an Elastizität fehlt, wirkt sie schnell faltig und meist wesentlich älter, als sie tatsächlich ist.

Um die Haut schnell zu beruhigen und den Hydrolipidfilm wieder in die natürliche Balance zu bringen, setzt BIODERMA beruhigende, rückfettende und restrukturierende Wirkstoffe in hochverträglichen Formeln ein. In kürzester Zeit wird trockene, raue Haut wieder seidig weich, richtig „streichelzart".

\section{Beruhigende Pflege auch für Babyhaut geeignet}

Atoderm-Produkte sind so sanft, dass sie sich auch für den Schutz und die Pflege von Kinder- und Babyhaut eignen. Denn auch die Kleinsten sollen sich rundum wohl in ihrer Haut fühlen!

Die Pflegeserie eignet sich für die tägliche Pflege der trockenen Haut, aber auch zur unterstützenden Behandlung bei Schüben, bei austrocknenden medikamentösen oder anderen Therapien sowie als Basispflege bei Neurodermitis oder Psoriasis.

Nach Informationen von Bioderma Laboratoire Dermatologique

\section{Sklerotherapie von Varizen}

\section{Erste europäische Leitlinienkonferenz}

\begin{abstract}
Die offiziellen Vertreter von 20 phlebologischen Gesellschaften aus ganz Europa wurden von der Deutschen Gesellschaft für Phlebologie eingeladen, um die erste europäische Leitlinie zur Sklerotherapie von Varizen aktiv mitzugestalten. Diese bisher in der Phlebologie einmalige Leitlinienkonferenz wird vom 8.6.-10.6.2012 in Mainz stattfinden.
\end{abstract}

Die Deutsche Gesellschaft für Phlebologie hat schon vor vielen Jahren eine Leitlinie zur Sklerosierungsbehandlung der Varikose erstellt. Diese Leitlinie wurde in vielen anderen europäischen
Ländern veröffentlicht und als aktueller Stand der Wissenschaft akzeptiert, da in den meisten Ländern keine nationalen Leitlinien zu diesem Thema existieren. Die von den nationalen Fachgesellschaf- ten entsandten Experten werden während der Leitlinienkonferenz eine einheitliche evidenzbasierte Leitlinie zur Sklerotherapie für den gesamten europäischen Raum verabschieden.

Dies soll der erste Schritt zu weiteren gemeinsamen europäischen Leitlinien im Fachgebiet Phlebologie sein und den Standard der phlebologischen Versorgung europaweit verbessern.

Nach Informationen der Deutschen Gesellschaft für Phlebologie 